\title{
Applications of platelet rich fibrin in dentistry
}

\author{
(D) Mamatha Shetty, (1) Srikala Bhandary
}

AB Shetty Dental College, Nitte University, Department of Periodontics, Karnataka, India

Date submitted:

31.12.2020

Date accepted:

01.03.2021

Online publication date: 15.03.2022

\section{Corresponding Author:}

Mamatha Shetty, M.D., AB Shetty

Dental College, Nitte University,

Department of Periodontics,

Karnataka, India

mamathadent99@yahoo.co.in

ORCID:

orcid.org/0000-0003-1092-0639

Keywords: Platelet, growth factors, periodontal regeneration, guided bone regeneration, soft tissue root coverage

\begin{abstract}
Platelet-rich fibrin (PRF), a second-generation platelet concentrate, comprises a fibrin matrix containing numerous growth factors. These growth factors are involved with the cells responsible for the processes of tissue repair, regeneration, and growth. The applications of PRF have surpassed those of other platelet concentrates such as platelet-rich plasma due to the ease and economical method of its preparation, as well the elimination of the need for supplemental exogenous compounds like bovine thrombin and calcium chloride during preparation. This brief communication highlights the applications of PRF in dentistry and medicine and focuses on its preparation method, as it involves low risks and satisfactory results with minimally invasive techniques.
\end{abstract}

\section{Introduction}

Platelet concentrates collected from the whole blood were first introduced in medicine more than 20 years ago. The first attempts to use concentrated platelet growth factors were derived from the knowledge that supra-physiological doses of these growth factors could be obtained from platelets to promote wound healing during and after surgery. The idea was later established into what is known today as platelet-rich plasma (PRP) which was introduced also in dentistry in the 1990s by several leading clinician-scientists such as Whitman and Marx $(1,2)$.

One of the main disadvantages of currently used biomaterials in tissue engineering is that the majority are typically avascular by nature, therefore, they do not provide the necessary vascular supply to provide successful regeneration of either soft or hard tissues $(1,3)$.
Platelets are the key components in the early phases of tissue regeneration as they release many growth factors, coagulation factors, adhesion molecules, cytokines/chemokines, and various other angiogenic factors that promote the proliferation and activation of cells involved in wound healing (4).

However, despite the growing success and use PRP of in the initial years, several limitations prevented its full potential. The technique of collection and processing was lengthy and therefore required the use of an additional anticoagulant such as bovine thrombin or $\mathrm{CaCl}_{2}$ (both known inhibitors of wound healing) to prevent clotting of the collected blood. These limitations brought the need to investigate new modalities for successful tissue regeneration $(2,5)$.

From this perspective, a second-generation platelet concentrate that does not require anticoagulants was developed, allowing a shorter preparation time, named platelet-rich fibrin (PRF) (6). 
Platelet concentrates are classified into pure plateletrich plasma, PRF, leukocyte and platelet-rich plasma, pure PRF, liquid PRF. The platelet concentrate obtained using the centrifugation process is used for regenerative treatment in periodontal disease.

\section{Preparation of PRF}

PRF was introduced as the first total autologous concept without additional anticoagulants. The lack of a need for an anticoagulant significantly reduces the risk of transcontamination (7). It also allows the physiological cell functions to continue after centrifugation (8). The main goal is to simplify the preparation process and minimize the required preparation steps and time for more suitable clinical applications. In this method, $10 \mathrm{~mL}$ peripheral venous blood is collected from a peripheral vein patient in a glass test tube. This is followed by immediate centrifugation at $3000 \mathrm{rpm}$ for $10 \mathrm{~min}$. If not immediately centrifuged, diffuse fibrin polymerization occurs, resulting in clot formation and reduction in quantity and quality (Figure 1) (1).

After processing PRF, the blood sample in the test tube is left to settle, allowing separation into three layers (8). The acellular plasma, or platelet-poor plasma, is the topmost straw-colored layer and, as the name suggests, lacks platelets. This step is followed by the PRF clot rich in fibrin and includes growth factors and cytokines in polymerized structure. The lowest, red fraction consists of erythrocytes.

When blood is collected in the test tube, it undergoes intrinsic coagulation due to contact with the glass, separating the blood into the clot and plasma.

During centrifugation, fibrinogen in the plasma fraction combines with the thrombin and forms the PRF region between the acellular plasma and the lower-packed red blood cell-rich fraction (8).

The superficial acellular fraction is removed, and the middle PRF fraction is collected using pliers along with the attached erythrocytes from the test tube. The fibrin clot is placed on a sterile surface and erythrocytes are scrapped off (Figure 2) (8).

Inflammatory cells and platelets are observed in abundance in the PRF matrices obtained by low-speed centrifugation force. Injectable PRF matrices also increase the number of platelets and leukocytes, which is obtained by low-speed centrifugation force (8).

\section{Uses of PRF in dentistry}

\section{A. Extraction socket management}

Extraction sockets have been managed using PRF as it is a natural fibrin matrix (8).

PRF can be used alone, replacing either a bone grafting material and/or barrier membrane. Since it is not necessary to use other biomaterials to cover an exposed flap, it offers the added advantage of exposing it to the oral cavity without risk of infection. PRF speeds the natural wound healing process without generating an immune response or foreign body reaction $(4,5)$.

PRF is typically stabilized by simply using an X-suture within the socket. Primary closure is not required. It has been shown that within a 3-month healing period, the fibrin matrix transforms into new tissue, bone in the socket with overlying soft tissue. The

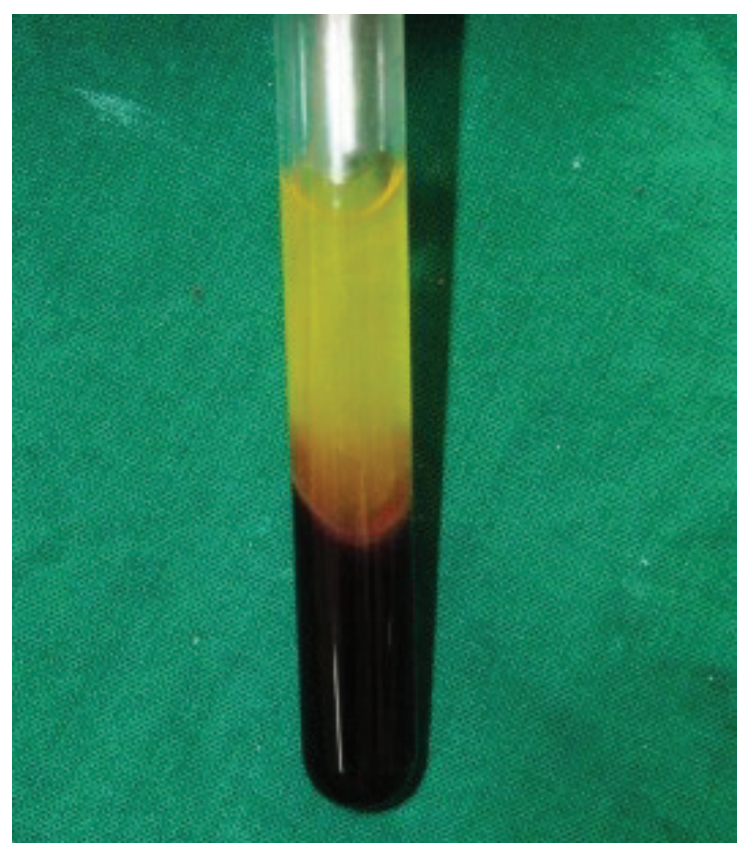

Figure 1. Test tube showing platelet rich fibrin after centrifugation of blood

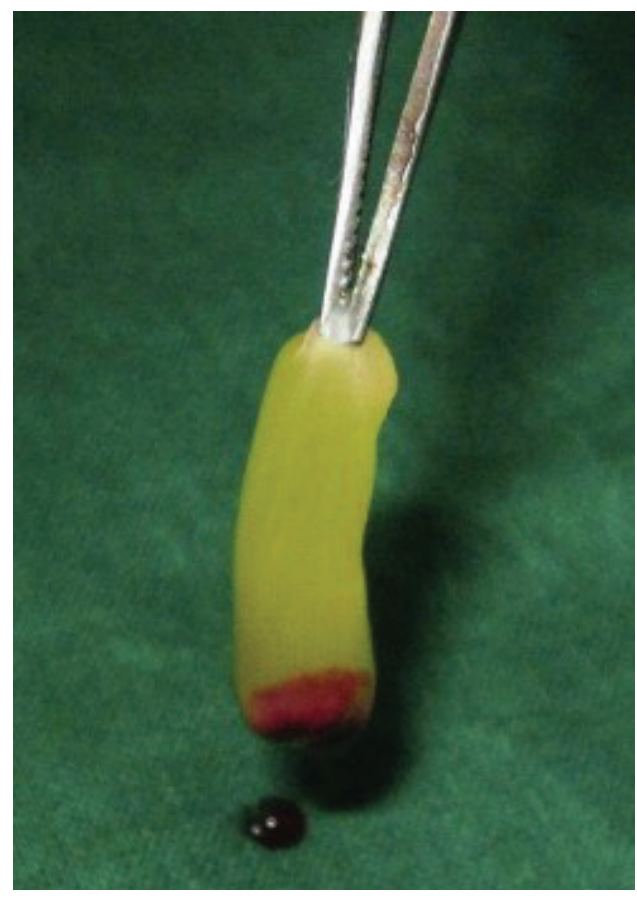

Figure 2. Platelet rich fibrin after centrifugation 
rationale is to apply pro-angiogenic, pro-inflammatory cytokines, and growth factors from PRF to stimulate healing in extraction sockets (8).

\section{B. Sinus elevation procedures}

PRF is used to repair the Schneiderian membrane, as sole grafting material and close the window during the lateral sinus approach (9). While the success rate of the above-mentioned procedures has been reported very high, very few comparative studies have been conducted so far (9). Others have shown that PRF could be combined with a bone grafting material for sinus lift augmentation to reduce the overall healing time (1).

\section{Soft tissue root coverage}

One of the other most widespread use of PRF has been reported for managing root exposure (10). Since PRF acts more directly on soft-tissue regeneration, numerous clinical studies have focused on the use of PRF during periodontal surgery of mucogingival defects. These studies have investigated the potential of PRF for soft-tissue management of Miller Class I and II defects (11). PRF can be used instead of connective tissue grafts in Miller Class I and II defects with a thick biotype, resulting in improved vascularization, wound healing, and patient morbidity (11). With a proper patient selection, PRF can be as effective as a connective tissue graft or using a collagen-derived xenograft material for Miller Class I and II recession defects (12). It improves wound healing and speeds the re-vascularization of tissues with similar root coverage without the need for a second surgical site from the palate or using a foreign body collagen membrane (10).

Millers Class I gingival recession can be effectively treated by coronally advanced flap with PRF or with a subepithelial connective tissue graft (10). Studies have shown better root coverage with the combination of subepithelial connective tissue graft and coronally advanced flap compared with the coronally advanced flap with PRF (10). PRF can be a better alternative in root coverage procedures, provided that they do not require a second surgical site.

\section{Periodontal regeneration}

The regeneration of periodontal tissues is much more complex than most tissues as it comprises many tissues/cell types from different embryonic origins (8).

The PRF matrix releases the growth factor slowly over an extended period, helping the regeneration process. PRF improves tissue repair and helps blood clot formation (8).

In periodontal diseases, the bone defect occurs due to bacteria and its byproducts, suggesting treatment with PRF can be useful since it contains leukocytes and macrophages capable of eliminating the pathogens.
PRF affects different types of cells like gingival fibroblasts, chondrocytes, osteoblasts, and endothelial cells by influencing their recruitment, proliferation, differentiation and helps in hard and soft tissue repair of the tissues (13).

\section{E. Guided bone regeneration}

Guided tissue regeneration (GTR) and guided bone regeneration (GBR) exhibit successful and predictable results (11). PRF can either be cut into small pieces and combined with various bone biomaterials/grafting materials or subsequently flattened and used as a barrier membrane in GTR/GBR procedures (12). It offers numerous advantages compared to traditional collagen membranes as it contains autologous growth factors and living host-immune leukocytes (12). These cells fight against incoming pathogens, reducing the rate of infection by as much as 10-fold (12). For this reason, PRF membranes bear the advantage in that they may be left exposed to the oral cavity without increased risk of contamination (14).

To date, there exist two methods to combine PRF with GBR procedures (14). The first acts as a barrier membrane, whereby the PRF scaffolds can be flattened into natural autologous barrier membranes with a resorption time of between 10 and 14 days and provides additional wound healing properties to the overlying soft tissues (14). The second aim is to supply bonegrafting particles with PRF by cutting PRF membranes into small "fragments" and mixing them with bone-grafting materials (14). The latter improves the handling properties of bone grafts by making them "stickier" but also provides the proteins and growth factors responsible for facilitating angiogenesis in bone biomaterials $(1,3)$.

\section{F. Implant placement and ridge augmentation}

A study on the effect of PRF placed in the maxillary posterior region and its association with implant stability after 4 to 6 weeks after implant placement showed that the implant placed with PRF showed better post insertion stability assessed by the resonance frequency analysis, compared with the contralateral region where implant placement was performed without PRF (15). The improved outcome was linked to the growth factors and the healing properties of PRF (16).

A review article concluded that, since PRF contains high number of growth factors, it has been used in healing extraction sockets, also in alveolar ridge augmentation procedures along with other bone grafts (16).

PRF is widely used by maxillofacial surgeons for the reconstruction of bone defects before implant placement. Evidence has shown improved osseous growth when PRF was used in intrabony defects (15).

PRF used in extraction sockets showed alveolar ridge preservation by decreasing alveolar width and height loss compared with the bone grafts used in alveolar sockets (16). 
Dhote and colleagues suggested that bone filling by PRF after enucleation of cyst followed by tooth extraction showed complete filling of bony defects (17).

A comparative study showed that PRF improved the preservation of the alveolar ridge, preventing bone resorption compared with the group in which no filling material was used (18). Studies have also shown successful closure of oral antral closure after extraction of maxillary first molar using PRF (19).

Another study that used PRF with and without flapless split crest in elderly patients showed positive results with PRF as it does not require the second stage surgery as well as improved healing and regenerative properties (20).

\section{The application of PRF in medicine}

PRF has been very useful in middle ear surgery as it helps a faster healing process, by accelerating cell proliferation and matrix remodeling (9).

PRF was shown to help angiogenesis and proliferation of preadipocytes, having a beneficial role in the consolidation of an adipocyte graft in the technique of facial aesthetic lipostructure procedure (21).

PRF can be used as an alternative to conventional myringoplasty as it has certain advantages like improved healing properties and reduced postoperative pain with minimal risk when used multiple times in a procedure (21). It was reported that treatment with PRF was successful when used for the repair of tympanic membrane perforations (21).

Topical application of PRF was used with inlay butterfly cartilage myringoplasty for tympanic perforation patients to assess healing of tympanic membrane and complications (22). The success rate of this technique improved with topical application of PRF (21). The autologous PRF not only enhances the healing of the graft but also protects it from infection (22).

In non-healing ulcers of patients with diabetes, which occurs due to various local and systemic factors, the autologous PRF matrix was found useful as it helped in painless healing of the wounds (23). PRF is a very economical and safe adjuvant to treat difficult wounds (23).

\section{Advantages (24)}

- No need for additional anticoagulants and chemicals are not required

- Favorable healing due to natural and slower healing

- Preparation protocol is standardized

- Cost-effectivity and simplicity

- PRF matrix has elasticity and flexibility properties that also help hemostasis

\section{Disadvantages (24)}

- The quantity of autologous blood samples and PRF is low

- Not useful for large wounds
- Need for quick handling after collection

- Storage impossible

- High antigenicity due to circulating immune cells (donor specific).

\section{Conclusion}

PRF offers the advantage of utilization and safely and efficiently delivery at supra-physiological doses of autologous growth factors to host tissues without the fear of a foreign body reaction or tissue rejection.

Regeneration of the periodontal tissues after treatment is a complex process as new cementum, periodontal ligament and alveolar bone are to be formed. The role of PRF in the formation of new tissues in the regeneration process needs to be confirmed by histological studies. It is crucial to assess the effect of PRF on soft and hard tissues based on histological evidence similar to well-researched recombinant protein. Another area of research is to determine whether the strength, stiffness, or toughness of PRF scaffolds should be modified via centrifugation protocols for the different clinical indications proposed.

\section{Ethics}

Peer-review: Externally peer-reviewed.

\section{Authorship Contributions}

Concept: M.S., Design: M.S., Data Collection or Processing: M.S., Analysis or Interpretation: M.S., Literature Search: S.B., Writing: S.B.

Conflict of Interest: No conflict of interest was declared by the authors.

Financial Disclosure: The authors declared that this study received no financial support.

\section{References}

1. Miron RJ, Choukroun J, eds. Platelet Rich Fibrin in Regenerative Dentistry: Biological Background and Clinical Indications. John Wiley \& Sons; 2017 Sep 12.

2. Marx RE. Platelet-rich plasma: evidence to support its use. J Oral Maxillofac Surg. 2004;62:489-496.

3. Zhang $\mathrm{Y}$, Zhang $\mathrm{X}$, Shi B, Miron RJ. Membranes for guided tissue and bone regeneration. Annals of Oral Maxillofacial Surgery. 2013;1:1-10.

4. Guo SA, Dipietro LA. Factors affecting wound healing. J Dent Res. 2010;89:219-229.

5. Saluja H, Dehane V, Mahindra U. Platelet-Rich fibrin: A second generation platelet concentrate and a new friend of oral and maxillofacial surgeons. Ann Maxillofac Surg. 2011;1:53-57.

6. Kobayashi E, Flückiger L, Fujioka-Kobayashi M, et al. Comparative release of growth factors from PRP, PRF, and advanced-PRF. Clin Oral Investig. 2016;20:2353-2360. 
7. Gupta V, Bains BK, Singh GP, et al. Regenerative potential of Platelet Rich Fibrin In Dentistry: Literature Review. Asian J Oral Health Allied Sci. 2011;1:22-28.

8. Agarwal M, Agrawal V. Platelet Rich Fibrin and its Applications in Dentistry-A Review Article. Natl J Med Dent Res 2014;2:5158.

9. Barbu HM, lancu SA, Hancu V, Referendaru D, Nissan $\mathrm{J}$, Naishlos S. PRF-Solution in Large Sinus Membrane Perforation with Simultaneous Implant Placement-Micro CT and Histological Analysis. Membranes (Basel). 2021;11:438.

10. Joshi A, Suragimath G, Varma S, Zope SA, Pisal A. Is Platelet Rich Fibrin a viable alternative to subepithelial connective tissue graft for gingival root coverage. Indian J Dent Resh. 2020;31:67-72.

11. Collins JR, Cruz A, Concepción E, López C, Hou W, Romanos GE. Connective Tissue Graft vs Platelet-rich Fibrin in the Treatment of Gingival Recessions: A Randomized Split-mouth Case Series. J Contemp Dent Pract. 2021;22:327-334.

12. Aroca S, Keglevich T, Barbieri B, Gera I, Etienne D. Clinical evaluation of a modified coronally advanced flap alone or in combination with a platelet-rich fibrin membrane for the treatment of adjacent multiple gingival recessions: a 6-month study. J Periodontol. 2009;80:244-252.

13. Mohan SP, Jaishangar N, Devy S, Narayanan A, Cherian D, Madhavan SS. Platelet rich plasma and platelet-rich fibrin in periodontal regeneration: A Review. J Pharm Bioallied Sci. 2019;11(Suppl 2):126-130.

14. Yoon JS, Lee SH, Yoon HJ. The influence of platelet rich. fibrin on angiogenesis in guided bone regeneration using xenogenic bone substitutes: A Study of rabbit cranial defects. J Craniomaxillofac Surg. 2014;42:1071-1077.

15. Tabrizi $\mathrm{R}$, Arabion $\mathrm{H}$, Karagah $\mathrm{T}$. Does platelet-rich fibrin increase the stability of implants in the posterior of the maxilla? A split-mouth randomized clinical trial. Int $\mathrm{J}$ Oral Maxillofac Surg. 2018;47:672-675.
16. Fan $\mathrm{Y}, \mathrm{Perez} \mathrm{K}$, Dym H. Clinical uses of platelet-rich fibrin in oral and maxillofacial surgery. Dent Clin North Am. 2020;64:291303.

17. Miron R, Choukroun J. Platelet Rich Fibrin in Regenerative Dentistry: Biological Background and Clinical Indications. John Wiley \& Sons Hoboken (NJ). 2017.

18. Canellas JVDS, Medeiros PJD, Figueredo CMDS, Fischer RG, Ritto FG. Platelet-rich fibrin in oral surgical procedures: a systematic review and meta-analysis. Int J Oral Maxillofac Surg. 2019;48:395-414.

19. Assad M, Bitar W, Alhajj MN. Closure of oroantral communication using platelet-rich fibrin: a report of two cases. Ann Maxillofac Surg. 2017;7:117-119.

20. Cortese A, Pantaleo G, Borri A, Caggiano M, Amato M. Platelet-rich fibrin (PRF) in implant dentistry in combination with new bone regenerative technique in elderly patients. Int $\mathrm{J}$ Surg Case Rep. 2016;28:52-56.

21. Shukla A, Kaurav YS, Vatsyayan R. Novel Use of Platelet Rich Fibrin Membrane in Transcanal Myringoplasty: A Prospective Study. Indian J Otolaryngol Head Neck Surg. 2020;72:355362.

22. Hosam M, Shaker M, Aboulwafa A. Effect of topical use of Platelet-Rich fibrin in repairing central tympanic membrane perforation using the endoscopic inlay butterfly cartilage myringoplasty technique. Egypt J Otolaryngol. 2017;33:557563.

23. Menon RR, Pillai AV, Shenoy V, Vijayaraghavan S. Autologous Platelet Rich Fibrin to treat difficult non-healing diabetic foot wounds with exposed bone-A Case report of this safe, effective and economical method. Amrita Journal of Medicine. 2020;16:181-184.

24. Borie E, Olivi DG, Orsi IA, et al. Platelet-rich fibrin application in dentistry: a literature review. Int J Clin Exp Med. 2015;8:79227929 . 